Sociohistórica, $n^{\circ}$ 41, e052, 1er. Semestre de 2018. ISSN 1852-1606

Universidad Nacional de La Plata.

Facultad de Humanidades y Ciencias de la Educación.

Centro de Investigaciones Socio Históricas

\title{
Saberes y prácticas de la psicología en la construcción histórica de la infancia y la familia.
}

\section{Reseña de Florencia Macchioli, Luciano García, Sebastián Benítez, Ana Briolotti, Gabriela Cardaci, Victoria Molinari, Itinerarios de la psicología. Circulación de saberes y prácticas en la Argentina del siglo XX, Buenos Aires, Miño y Dávila, 2017, 238 páginas}

\section{Leandro Stagno *}

* Facultad de Humanidades y Ciencias de la Educación - Universidad Nacional de La Plata, Argentina

leandrostagno@yahoo.com.ar

Cita recomendada: Stagno, L. (2018). Saberes y prácticas de la psicología en la construcción histórica de la infancia y la familia. Sociohistorica, 41, e052. https://doi.org/10.24215/18521606e052 


\title{
Saberes y prácticas de la psicología en la construcción histórica de la infancia y la familia.
}

\author{
Reseña de Florencia Macchioli, Luciano García, Sebastián Benítez, Ana Briolotti, Gabriela Cardaci, \\ Victoria Molinari, Itinerarios de la psicología. Circulación de saberes y prácticas en la Argentina del siglo \\ XX, Buenos Aires, Miño y Dávila, 2017, 238 páginas
}

Leandro Stagno

Facultad de Humanidades y Ciencias de la Educación -

Universidad Nacional de La Plata, Argentina

leandrostagno@yahoo.com.ar

En una revisión bibliográfica recientemente publicada, Eduardo Zimmermann señala que las perspectivas globales o transnacionales han ocupado un lugar central en la producción historiográfica. Según sus precisiones, el concepto transnacional en estas investigaciones remite de manera simultánea a las características de sus objetos de estudio y al prisma desde el cual se los analiza, dado que el foco está puesto en los movimientos, los flujos y la circulación de personas, saberes y bienes materiales y simbólicos a través de fronteras nacionales y culturales. Zimmermann fundamenta sus argumentos en una exhaustiva reseña de investigaciones que han aportado al conocimiento de las historias políticas nacionales y regionales, la circulación de saberes, los procesos de constitución de campos académicos y científicos, y al de las dinámicas de internacionalización de las elites (Zimmermann, 2017).

El libro que aquí se reseña se inscribe en dicha producción y recupera la citada duplicidad de lo transnacional para estudiar la circulación de saberes y prácticas de la psicología entre diferentes fronteras nacionales e institucionales, en una escala que enfatiza las dinámicas propias de la Argentina del siglo XX. Los seis estudios reunidos recuperan los avances o los resultados de investigaciones desarrolladas en el marco del Programa de Estudios Históricos de la Psicología en la Argentina, con sede en la Facultad de Psicología de la Universidad de Buenos Aires. Este espacio ha sido pionero en el estudio de estas temáticas, y ha nucleado a investigadores e investigadoras tanto de la referida institución como de su homónima en la Universidad Nacional de La Plata, y también del Consejo Nacional de Investigaciones Científicas y Técnicas. Esta inscripción explica la sinergia establecida con el libro que Luciano García, Florencia Macchioli y Ana María Talak editaron en 2014, que resulta un aporte ineludible a la hora de estudiar la historia de la producción del conocimiento psicológico sobre la infancia, la adolescencia y la familia (García, Macchioli y Talak, 2014).

El capítulo escrito por Sebastián Benítez analiza los artículos publicados en El Monitor de la Educación Común -órgano de difusión del Consejo Nacional de Educación-referidos al desarrollo moral, afectivo y físico de los niños y las niñas, a fin de comprender el modo en que los saberes psicológicos fundamentaron las formas de pensar la infancia escolarizada en tiempos de la configuración del sistema educativo nacional. Por un lado, remite a las demandas formuladas a la escuela y a la familia en favor de propiciar un desarrollo infantil acorde a los parámetros de normalidad estipulados; por otro lado, reseña las argumentaciones asociadas a definir cuáles eran los juegos apropiados para los niños y cuáles para las niñas, en tanto opciones excluyentes. Tal como señala Benítez, ambas intervenciones ofrecían fundamentos para identificar desviaciones y para definirlas en términos de déficit y anormalidad. 
El segundo capítulo, a cargo de Victoria Molinari, recurre a la citada revista para estudiar la producción, traducción y difusión de saberes sobre el nivel intelectual infantil en los ámbitos educativos durante las primeras tres décadas del siglo XX. El Monitor de la Educación Común expuso los debates que en Europa y Estados Unidos enfrentaban a quienes se interesaban por variables relacionadas con la herencia o por las condiciones ambientales tanto como las iniciativas argentinas que retomaban estas posturas y que, como en dichas latitudes, tampoco cuestionaban el papel conferido a la raza a la hora de estudiar el nivel intelectual infantil. Según Molinari, las explicaciones basadas en la antropometría dieron paso a otras en las que predominó el recurso a escalas métricas fundamentadas en cuantificaciones estadísticas. Esta modificación estuvo acompañada por conceptualizaciones sobre la inteligencia basadas en dinámicas ambientales y sociales que relegaron las explicaciones fundadas en la raza y el fenotipo.

Ana Briolotti se ocupa de la circulación de saberes de la psicología en el ámbito de la medicina infantil entre las décadas de 1930 y 1960, en un análisis que focaliza en las reflexiones sobre el componente psíquico de las enfermedades contraídas por los lactantes en la institución hospitalaria donde estaban internados. La autora demuestra que las tesis del psiquiatra y psicoanalista inglés John Bowlby introdujeron cambios en torno a la comprensión de esta patología, definida desde finales del siglo XIX como hospitalismo. El informe que Bowlby redactó en 1950 para la Organización Mundial de la Salud no solo atendía a la disposición espacial de los hospitales y a la asepsia de las salas, sino que también refería a la necesidad de garantizar el afecto y los cuidados maternos, en línea con anteriores intervenciones forjadas en el ámbito de la pediatría que procuraban limitar los tiempos de internación y sostener el lazo materno-filial. Briolotti demuestra que estas tesis se proyectaron sobre la renovación de la pediatría y de las pautas de crianza alentadas en la Argentina de los años sesenta por Eva Giberti y Florencio Escardo. Sus reflexiones instaban a evitar "la mutilación institucional de la familia" y, más allá de las situaciones de internación, vinculaban la salud mental de los niños y las niñas con los cuidados brindados durante la primera infancia.

En el cuarto capítulo, Luciano García reconstruye las condiciones que permiten explicar la legitimidad conferida en la Argentina a la psicología del desarrollo de Henri Wallon, médico y filósofo francés ligado a la Internacional Comunista. En particular remite a aquellas que tornaron viable la traducción local de su obra al castellano. El análisis proseguido entiende este proceso de traducción como parte de una cultura comunista que encontraba en los saberes científicos un instrumento para intervenir políticamente en la coyuntura abierta por las guerras mundiales. Por un lado García demuestra que quienes difundieron sus ideas en la Argentina eran intelectuales que adherían a las causas filosoviéticas, antifascistas y comunistas, que se habían vinculado con Wallon en Francia, que discutían su obra y la interpretaban a la luz de temas y problemas de la psicología, la psiquiatría, el psicoanálisis y la educación, y que prologaban las traducciones publicadas a instancias de editoriales organizadas por el Partido Comunista Argentino. Por otro lado, el autor describe dos momentos en la publicación de las referidas traducciones que coinciden con cambios en el interior del comunismo argentino e internacional. Uno inicial a mediados de la década de 1940, cuando los y las intelectuales comunistas tenían una denodada gravitación en la arena política internacional, otro de declive, asociado a la crisis internacional y local del comunismo y a aquella que enfrentó la industria editorial en la Argentina en tiempos de la última dictadura.

Florencia Macchioli estudia la implementación de la terapia familiar en Buenos Aires en los años sesenta y los tempranos setenta, desde la óptica de la circulación local de los aportes estadounidenses e italianos. La autora explica que el inicio del abordaje familiar en Estados Unidos fue propiciado por investigaciones centradas en la comunicación y sus perturbaciones, y que en Italia las psicopatologías que llevaron al interés por este tipo de terapéutica fueron los trastornos alimenticios, las fobias, las toxicodependencias, las enfermedades psicosomáticas y los cuadros borderlines. Este prisma analítico permitió a Macchioli identificar dos momentos en el origen del abordaje familiar en el contexto argentino. El primero estuvo representado por la conjunción de las acciones desplegadas en el Servicio de Psicopatología del Hospital "Aráoz Alfaro" de Lanús, las comunicaciones propiciadas desde la revista Acta Psiquiátrica y Psicológica de América Latina, y la 
intervención profesional de Carlos Sluzki, lector y traductor de los aportes sistémicos de Estados Unidos en el despuntar de los años sesenta. El segundo momento coincide con la publicación de Terapia Familiar, revista creada y editada por Alfredo Canevaro, quien abrió canales de comunicación con los desarrollos italianos y lideró la promoción local de la especialidad cuando Sluzki se radicó en Estados Unidos.

El libro cierra con el capítulo escrito por Gabriela Cardaci, en el que reconstruye la trama intelectual gestada en torno a Lo Grupal, revista publicada entre 1983 y 1993 con la dirección de Eduardo Pavlovski y Juan Carlos De Brasi. Sus argumentos iluminan dos aspectos de la producción de conocimientos en torno al abordaje de lo grupal. Uno de ellos remite a las experiencias de trabajo realizadas por los articulistas y los directores de la revista durante sus exilios, y a las vías de intercambio internacional suscitadas a partir de esta experiencia. El otro describe el cuestionamiento a los modelos teóricos de la clínica psicológica y psicoanalítica que, en términos generales, procuraba reafirmar la relación entre la práctica profesional, la intelectual y la política. Como sostiene Cardaci, en este proyecto editorial confluían discursos que problematizaban el papel de las dinámicas sociohistóricas en las prácticas de salud metal y, en este sentido, instaban a interrogar la relación entre la clínica y la cuestión política.

En términos de balance, dos decisiones interpretativas asumidas por los autores y las autoras son las que confieren unidad al libro. Una de ellas supuso estudiar la consolidación de redes científicas transnacionales a fin de explicar el fortalecimiento y difusión de los saberes y las prácticas de la psicología en la Argentina. Las dinámicas de estas redes son reconstruidas en cada uno de los capítulos a partir del análisis de políticas editoriales, intercambios gestados a instancias de congresos internacionales, viajes de estudio y aquellos asociados a la experiencia del exilio. La otra decisión consistió en comprender el papel que cumplieron los saberes y las prácticas "psi" en la configuración de modalidades de intervención sobre las infancias y las familias en el correr del siglo XX. La familia nuclear es presentada como entorno de crianza ideal, promotora del desarrollo infantil y aliada de la institución escolar; el grupo familiar es interpelado en tanto objeto de una terapéutica que procuraba encontrar respuestas a las preguntas por acotadas patologías. Por cierto, la constatación de estas regularidades permite sugerir diálogos entre el libro reseñado y específicos antecedentes de investigación forjados en tres campos de estudio: la historia de la educación, la historia de la familia y la historia de la infancia.

La historia de la educación en la Argentina ha abordado la configuración de espacios de producción de conocimientos que, sostenidos en los desarrollos de la psicología experimental, procuraban clasificar y calificar el desarrollo infantil y consolidar a la escuela moderna en tanto tecnología apropiada para la transmisión de saberes y moralidades (Dussel, 2014; Finocchio, 2009; Puiggrós, 1990; Southwell, 2003; Suasnábar y Palamidessi, 2006). En otras latitudes se ha advertido sobre la potencia de estudiar los congresos internacionales a la hora de conocer la circulación de ideas pedagógicas, de modelos de enseñanza y de movimientos de reforma educativa durante el siglo XX, procesos donde han intervenido saberes y prácticas de la psicología (Brehony, 2004; Fuchs, 2004).

El campo de los estudios históricos de la familia en la Argentina se encuentra en plena expansión. La tensión entre la heterogeneidad de las prácticas familiares y una normatividad que las excluía, la conformación de un modelo familiar basado en la pauta nuclear y sus posteriores cuestionamientos, la difusión de discursos expertos sobre maternidad y paternidad, las políticas de Estado vinculadas a fortalecer el binomio madre-hijo son temas que forman parte del citado campo de estudio y dialogan de cerca con el conjunto de los capítulos del libro que se reseña (Cosse, 2006, 2008, 2010; Nari, 2004).

El tercero de los campos aludidos es el de la historia de la infancia. La complejización de los temas que desde la década de 1990 comenzaron a integrar su agenda da cuenta de la consolidación y expansión de espacios académicos que tomaron a las infancias como objeto específico de indagación histórica, tanto en las escalas nacionales como en las regionales y transnacionales (Zapiola, en prensa). Para la Argentina, dos compilaciones editadas en 2010 y en 2011 expresan la profusión de estas indagaciones y traen estudios 
específicamente referidos a los discursos "psi” (Cosse, Llobet, Villalta y Zapiola, 2011; Lionetti y Míguez, 2010).

Una de las aristas de la revisión bibliográfica citada al inicio de este escrito permite darle cierre. Más que constatar la difusión de teorías científicas, ideologías políticas o tendencias culturales desde Europa y Estados Unidos hacia el resto del mundo, Eduardo Zimmermann llama a reconocer sus transformaciones en momentos de adaptarse y generalizarse en otro contexto, es decir, se trata de comprender la configuración de nuevos conocimientos a la luz de la hibridación operada en el tránsito entre diferentes contextos. Esta apuesta interpretativa no ha sido el eje del libro reseñado, cuyos capítulos refieren centralmente a los procesos de recepción local de saberes y prácticas de la psicología. Quienes quieran asumir este desafío encontrarán sustantivos aportes en las investigaciones de Florencia Macchioli, Luciano García, Sebastián Benítez, Ana Briolotti, Gabriela Cardaci y Victoria Molinari, desde donde podrán conocer, además, aspectos claves de algunos de los "itinerarios de la psicología".

\section{REFERENCIAS BIBLIOGRÁFICAS}

Brehony, K. (2004). A New Education for a New Era: The Contribution of the Conferences of the New Education Fellowship to the Disciplinary Field of Education, 1921-1938. Paedagogica Historica, 40(5-6), 733-755.

Cosse, I. (2006). Estigmas de nacimiento. Peronismo y orden familiar, 1946-1955. Buenos Aires: Fondo de Cultura Económica.

Cosse, I. (2008). Presentación del dossier. La historia de la familia en la Argentina del siglo XX: nuevas perspectivas de un campo en construcción. Anuario IEHS, 23, 343-353.

Cosse, I. (2010). Pareja, sexualidad y familia en los años sesenta. Una revolución discreta en Buenos Aires. Buenos Aires: Siglo XXI.

Cosse, I., Llobet, V., Villalta, C., y Zapiola, M. C. (ed.) (2011). Infancias: politicas y saberes en Argentina y Brasil. Siglos $X I X y X X$. Buenos Aires: Teseo.

Dussel, I. (2014). Víctor Mercante y la producción de un discurso científico sobre la educación. Archivos de Ciencias de la Educación, 8(8), 1-15.

Finocchio, S. (2009). La escuela en la historia argentina. Buenos Aires: Edhasa.

Fuchs, E. (2004). Educational Sciences, Morality and Politics: International Educational Congresses in the Early Twentieth Century. Paedagogica Historica, 40(5-6), 757-784.

García, L., Macchioli, F., y Talak, A. M. (2014). Psicología, niño y familia en la Argentina, 1900-1970. Perspectivas bistóricas y cruces disciplinares. Buenos Aires: Editorial Biblos.

Lionetti L., y Míguez, D. (comp.) (2010). Las infancias en la historia argentina. Intersecciones entre prácticas, discursos e instituciones (1890-1960). Rosario: Prohistoria.

Nari, M. (2004). Politicas de maternidad y maternalismo politico. Buenos Aires (1890-1940). Buenos Aires: Biblos.

Puiggrós, A. (1990). Sujetos, disciplinas y currículum en los orígenes del sistema educativo argentino (1885 - 1916). Buenos Aires: Galerna.

Southwell, M. (2003). Psicología Experimental y Ciencias de la Educación. Notas de historias y fundaciones. La Plata: EDULP.

Suasnábar, C., y Palamidessi, M. (2006). El campo de producción de conocimientos en educación en la Argentina. Notas para una historia de la investigación educativa. Historia de la Educación. Anuario, 7, 6-40.

Zapiola, M. C. (en prensa). Estado e infancia en Argentina: reflexiones sobre un recorrido historiográfico. En AA.VV., Historia de la infancia en América Latina: discusiones, trayectorias y desafios. Red de Estudios de Historia de las Infancias en América Latina. Universidad Nacional del Centro de la Provincia de Buenos Aires.

Zimmermann, E. (2017). Una nota sobre nuevos enfoques de historia global y transnacional. Estudios Sociales del Estado, 3(5), 12-30. 